\title{
Self-imaging of spin waves in thin, multimode ferromagnetic waveguides
}

\author{
Mateusz Gołębiewski*, ${ }^{*}$, Paweł Gruszecki* and Maciej Krawczyk* \\ *Institute of Spintronics and Quantum Information, Faculty of Physics, \\ Adam Mickiewicz University in Poznań, Uniwersytetu Poznańskiego 2, 61-614 Poznań, Poland \\ ${ }^{\dagger}$ matgol2@amu.edu.pl
}

\begin{abstract}
Self-imaging of waves is an intriguing and spectacular effect. The phenomenon was first observed for light in 1836 by Henry Fox Talbot and to this day is the subject of research in many areas of physics, for various types of waves and in terms of different applications. This paper is a Talbot-effect study for spin waves in systems composed of a thin, ferromagnetic waveguide with a series of single-mode sources of spin waves flowing into it. The proposed systems are studied with the use of micromagnetic simulations, and the spin wave self-imaging dependencies on many parameters are examined. We formulated conditions required for the formation of self-images and suitable for experimental realization. The results of the research form the basis for the further development of self-imaging-based magnonic devices.
\end{abstract}

Index Terms - magnonics, spin waves, Talbot effect, self-imaging, waveguides.

\section{INTRODUCTION}

Due to their unique properties, such as a strong dependence on the material parameters and the magnetization orientation in relation to the direction of propagation or the film plane, spin waves (SWs, i.e, coherent magnetization disturbances propagating in magnetic materials in the form of waves) are a fascinating research object[1]. Their dynamic properties are related to coexisting short-range, strong, and isotropic exchange interactions and long-range, weak, and anisotropic magnetostatic interactions. Moreover, one of the essential advantages of SWs as an information carrier is the fact that their frequency spans range from a few to hundreds of gigahertz, with the corresponding wavelength range extending from micrometers to tens of nanometers. All this adds up to the type of medium that can be influenced and configurable on many levels, making them flexible for applications in devices that can potentially support or replace conventional electronic solutions [2], [3].

Usually, the transmission and processing of SWs in narrow waveguides [4], [5] that are often coupled[6] are considered in magnonics. Another promising line of research is the use of elements much wider than the waveguides themselves to redirect[7, [8, 9] and process SWs [10, [11, [12], [13, [14. These elements, being multimode waveguides, can be referred to as processing blocks. Most commonly, SWs are delivered to these elements via a single or a group of narrow waveguides. Subsequently, these entering SWs interfere with each other. Moreover, this interference can be further molded in processing blocks by properly modifying the medium where SWs propagate. It can be achieved by the introduction of defects 14, programmable magnetic elements on top of that region[12, or the utilization of noncolinear magnetization textures[15]. The main advantage of this approach is the possibility of using interference effects to process SW-carried information[12], [13, [14].

It is important to note that as waves fall into the processing block from a group of evenly spaced waveguides, the interference image with the same symmetry as the waveguide array is formed. There is a strong analogy to the Talbot effect [16], 17] that was firstly observed for electromagnetic waves. It results from waves interference coming from a series of periodically arranged sources. The most visible distribution of self-images, the so-called Talbot carpet, is created for infinitely many sources, corresponding to an infinitely long diffraction grating through which a plane wave passes. This phenomenon is extensively studied in recent years for many types of waves 18 and found already applications, for instance to improve x-ray imaging[19]. It has been theoretically demonstrated that this effect can occur also for SWs 20]. However, the conditions of the formation of the SWs' self-images in a thin ferromagnetic multimode waveguide have never been studied.

In this paper, we numerically investigate prototypes of SW multimode waveguides with multiple input single-mode stripes (see Fig. 2) and use the phenomenon of multimode interference (MMI). The fact that self-imaging is here an inherent property of the system allows assuming that in this type of device the interference images will form patterns analogous to the classic Talbot carpets. We will analyze how the width of the multimode waveguide, the number and separations between input waveguides influence the interference pattern, and finally we will discuss prospects for applications. We believe that due to the advantage of magnonic systems over the photonic ones in terms of miniaturization, the self-imaging effect may result in efficient magnonic devices, such as logic gates, couplers, multi/demultiplexers, or phase controllers.

The paper is organized as follows. In Section [II we describe system and method used in our investigations. In Section III we present the simulation results for different geometrical parameters and in Section IV] we discuss the influence of damping on formation of the self-images. The paper is finishing with conclusions of the results. 


\section{MODEL DESCRIPTION}

\section{A. Self-imaging}

The phenomenon of the self-imaging of waves is well described and widely used today 18, 21. It dates back to the early 19th century when Henry Fox Talbot first observed it for light passing through a diffraction grating and a lens with high magnifying power 16 .

The Talbot effect is the result of waves' interference as they pass through any periodic aperture. In its close diffraction field, at strictly defined distances from the sources, replication of the periodic structure - self-imaging will take place [see Fig. 1(a)]. The effect is the more visible, the wider diffraction grating is in relation to the wavelength of waves passing through it. For an infinitely long periodic grating (or sources array), one can obtain the Talbot carpet - ideal and repeated recreations of the periodic structure in every period, so-called Talbot lengths, described by a formula

$$
z_{\mathrm{T}}=n d^{2} / \lambda
$$

where $n$ is an integer specifying the number of subsequent self-images, $d$ is a period of the grating, and $\lambda$ is a wavelength. The theory of the Talbot effect has been described many times for various types of interactions [20], so in this paper, we will limit it only to the general description. (a)

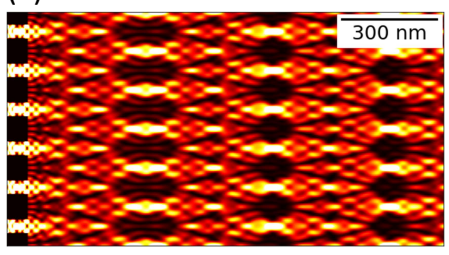

(b)

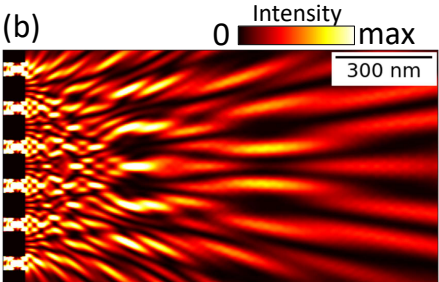

Figure 1. Illustrative self-imaging representation for (a) an infinitely long array of sources on the left and (b) for a finite number of inputs, where the near diffraction field quickly turns into the far diffraction field, and the Talbot effect disappears. In both cases, SW frequency is equal to $40 \mathrm{GHz}$, inputs period is $120 \mathrm{~nm}$ and infinitely wide waveguides were assumed.

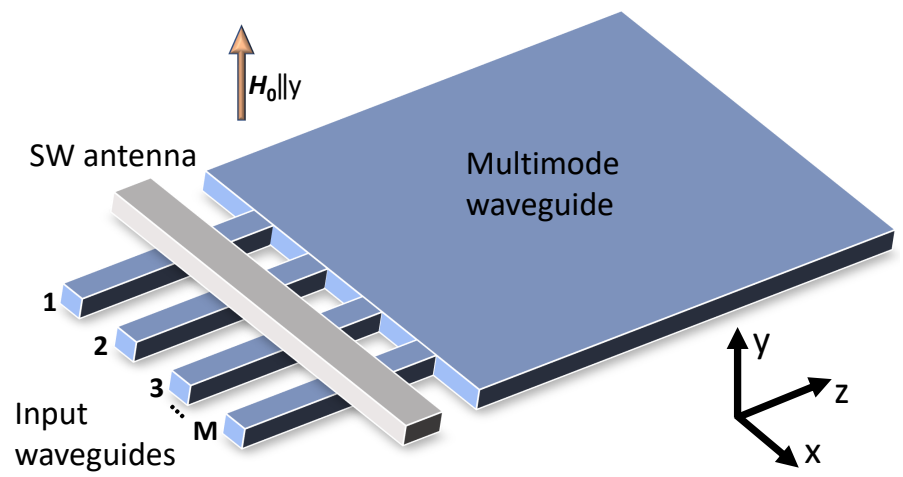

Figure 2. Scheme of a $M$-input multimode device.

\section{B. Micromagnetic simulations}

In order to present magnonic systems based on the self-imaging effect with MMI, the micromagnetic package
MUMAX3 was used 22. The studied systems were discretized uniformly by $5 \times 5 \times 5 \mathrm{~nm}^{3}$ unit cells, with one cell per thickness. The following magnetic parameters were applied in the simulations: saturation magnetization of $M_{\mathrm{S}}=860 \mathrm{kA} / \mathrm{m}$, an exchange constant of $A=13 \mathrm{pJ} / \mathrm{m}$, these are the parameters related to permalloy, and initially negligibly low damping constant $\alpha=10^{-5}$. In each of the analyzed cases, the waveguide was homogeneously magnetized by the external magnetic field of the value $\mu_{0} H_{0}=1.1 \mathrm{~T}\left(H_{0}>M_{\mathrm{S}}\right)$, directed perpendicular to the plane of the system. Low amplitude harmonic SWs were excited continuously by a microwave antenna placed on each of the input single-mode waveguides of $40 \mathrm{~nm}$ width, at a distance of $90 \mathrm{~nm}$ from the multimode part (see Fig. 2), until the system reached a steady state, i.e. a state where the interference image in the multimode layer is no longer dependent on time. The analyzed frequency of the SWs in our systems is $40 \mathrm{GHz}$ enabling to excite exchange interaction dominated SWs of the wavelength $(\lambda=68.83 \mathrm{~nm})$ only a dozen times greater than the exchange length $\left(l_{\mathrm{ex}}=\sqrt{2 A /\left(\mu_{0} M_{\mathrm{S}}^{2}\right)}=6 \mathrm{~nm}\right)$. To illustrate the propagation of SWs through the system, the intensity maps were calculated as the square of the $m_{x}$-component of magnetization and averaged over time $\left\langle m_{x}^{2}\right\rangle_{t}$ after reaching the steady state.

\section{Multiple, LONG-RANGE SELF-IMAGING IN MULTIMODE WAVEGUIDES}

The simulations were divided into three parts, where the Talbot effect in the $M$-input MMI systems was examined in terms of parameters like the distance between the inputs, the number of inputs, and the multimode waveguide width. In each of the presented cases, the impact of changing only one of these parameters was analyzed, leaving the other unchanged. This allows to access the possibilities of manipulating interference images in terms of their best use in future magnonic devices.

1) Variable distance between inputs: The first studies are for systems with a variable distance between the inputs while maintaining a constant number of them and unchanged width of the system. We assume $M=8$ inputs and the waveguide width equal $1.64 \mu \mathrm{m}$.

The analysis of the SW interference images in the multimode layer in Fig. 3 clearly shows that the interference first causes the formation of self-images in the near diffraction field, then due to the MMI, the structure is reconstructed further away from the source. As the distance between the inputs increases, so does the distance at which the reproductions of Talbot carpets appear. By further increasing the distance between the inputs while keeping the condition of their number and system width constant, we will come to the point where the series of inputs will span the entire width of the waveguide (see Fig. 4).

For large distances, when the inputs fill whole waveguide width, it can be noticed, that in near field the self-imaging effect is very clear. The first Talbot images (a series of first reproductions of periodic sources, laterally shifted in phase 

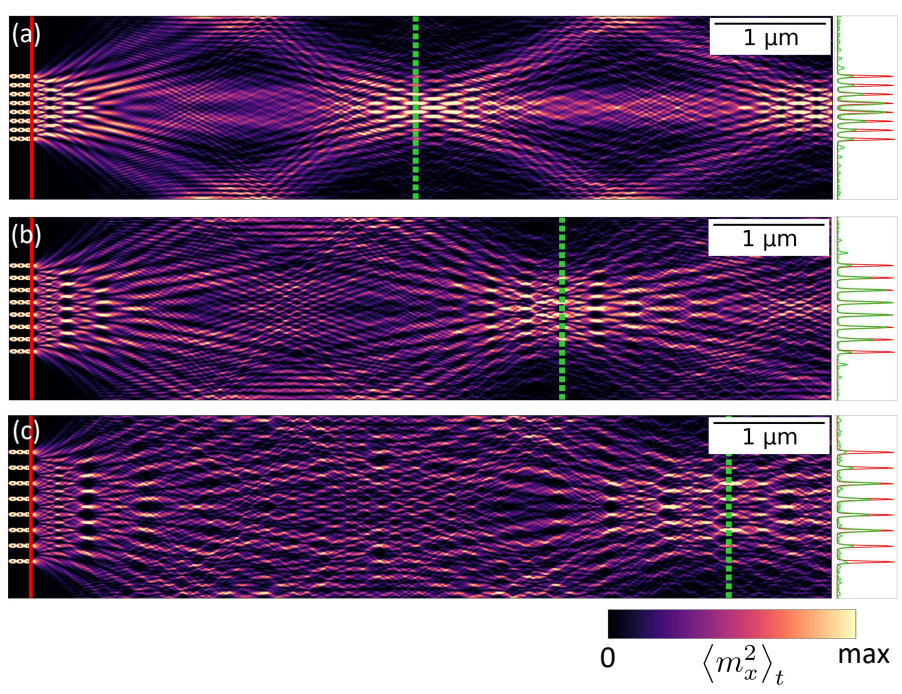

Figure 3. Intensity maps of SWs entering from a series of 8 single-mode $40 \mathrm{~nm}$ wide waveguides (on the left) into a $1.64 \mu \mathrm{m}$ wide multimode film. The distance between the sources is respectively (a) $80 \mathrm{~nm}$, (b) $110 \mathrm{~nm}$, and (c) $140 \mathrm{~nm}$. The solid red and dashed green vertical lines represent the cross-section along which the normalized SW intensities as a function of the width are plotted (on the right). (a)

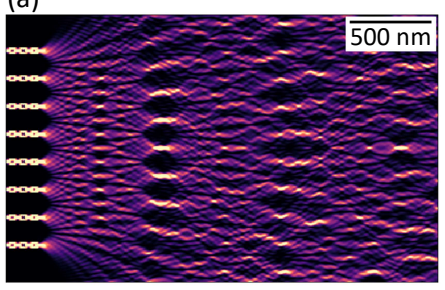

(b)

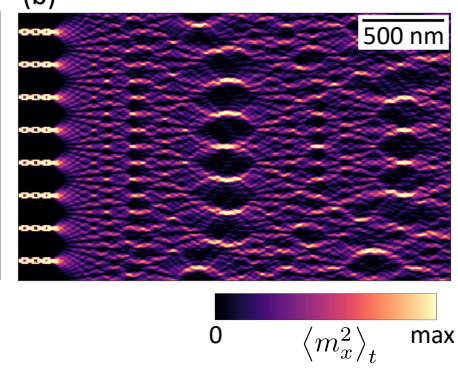

Figure 4. Intensity maps of SWs in the near diffraction field of multimode, $1.64 \mu \mathrm{m}$ wide waveguides, with 8 inputs separated by (a) $170 \mathrm{~nm}$ and (b) $200 \mathrm{~nm}$.

by half of their period) are very similar to the theoretical ones [see Fig. 1 (a)], despite the finite dimensions of the system. The difference is only visible at the edges of the system, where the resulting SW focus points are clearly out of line with the others, disturbing the self-imaging. For the first two Talbot lengths (see Eq. 1), this disturbance does not significantly affect the phenomenon, however, for further distances the pattern regularity is increasingly disturbed. Nevertheless, due to reflections from the waveguide edges, the reproductions of the entire patterns are created also at a distance of a dozen $\mu \mathrm{m}$. However, the self-images resulting from the Talbot effect in the near field are more interesting from the application point of view, because they maintain their regularity and high intensity (see the insets on the right side of Fig. 3), even in materials with relatively high damping, as will be discussed in Section IV

2) Variable number of inputs: The second type of numerical simulation is performed to check how the change in the number of single-mode inputs affects the formation of both Talbot carpets and their reproductions on the further sections of multimode waveguide. Analogically to the previous paragraph, the width of the system and the distance between the sources will remain unchanged here, i.e., $80 \mathrm{~nm}$ and $1.64 \mu \mathrm{m}$, respectively.

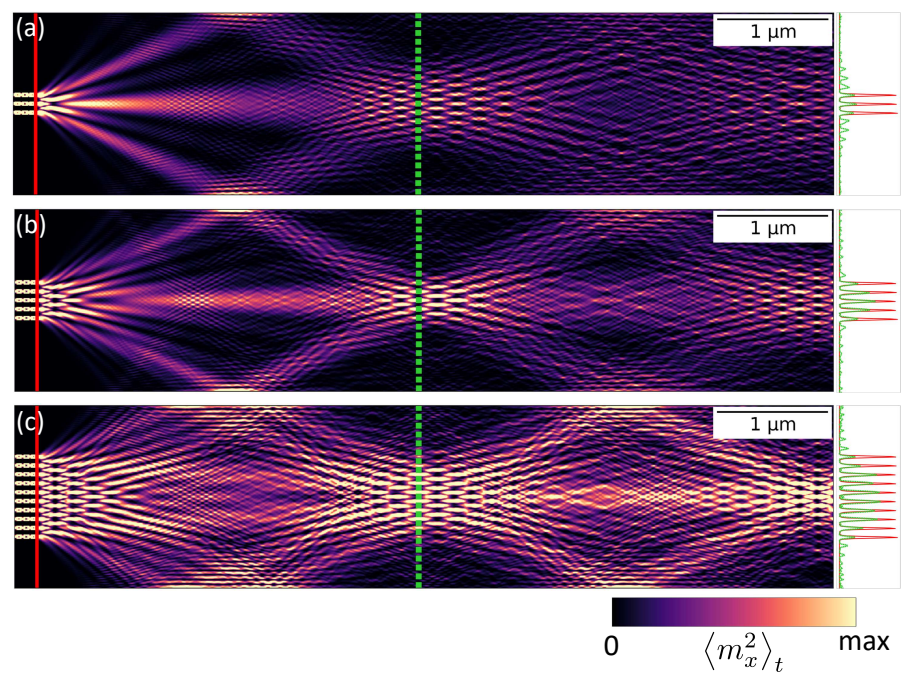

Figure 5. Intensity maps of SWs in a $1.64 \mu \mathrm{m}$ wide multimode film coming from (a) 3, (b) 5, and (c) 10 single-mode inputs, separated by $80 \mathrm{~nm}$. The solid red and dashed green lines represent the cross-section along which the normalized SW intensities as a function of the width are plotted (on the right).

In Fig. 5 it can be seen that, in contrast to the distance between the inputs, their number does not affect the distance at which the Talbot pattern is reconstructed in a multimode waveguide. However, for a small number of sources, this selfimaging in far field practically does not occur - for three sources in Fig. 5(a), a specific interference pattern can be seen, but with significantly lower intensity. Only in the case of 5 or more sources, we can observe the reproduction of the near diffraction field.

3) Variable $M M I$ waveguide width: The last type of system in which we decided to analyze SW self-imaging are multimode waveguides with different widths. Here again, as in the previous cases, the remaining parameters tested, i.e., the number and the distance between the inputs, are constant, $M=10$ and $80 \mathrm{~nm}$, respectively.

The simulation results presented in Fig. 6 clearly show, following the intuition from geometric optics, the tendency of the SWs' re-focus area to move away from the inputs with increasing width of the waveguide. It points that the width of the multimode waveguide and its edges are important factors influencing reproduction of the pattern in far field. Comparing Figs. 6(a)-(c), it can be seen that the interference image shown in Fig. 6(a) most closely resembles the classical Talbot image, a similar conclusion may be drawn from comparing Fig. 3(a)-(c). This indicates that by properly choosing the width of the multimode waveguide, the number and a separation between the input single-mode waveguides, one can reproduce a Talbot carpet in relatively far distances in multimode waveguides, the same as for an infinite number of periodically arranged SW sources. This is because, the lateral edges on which reflections occur can act similarly to periodic boundary conditions. This means that for materials with low damping, it can be possible 


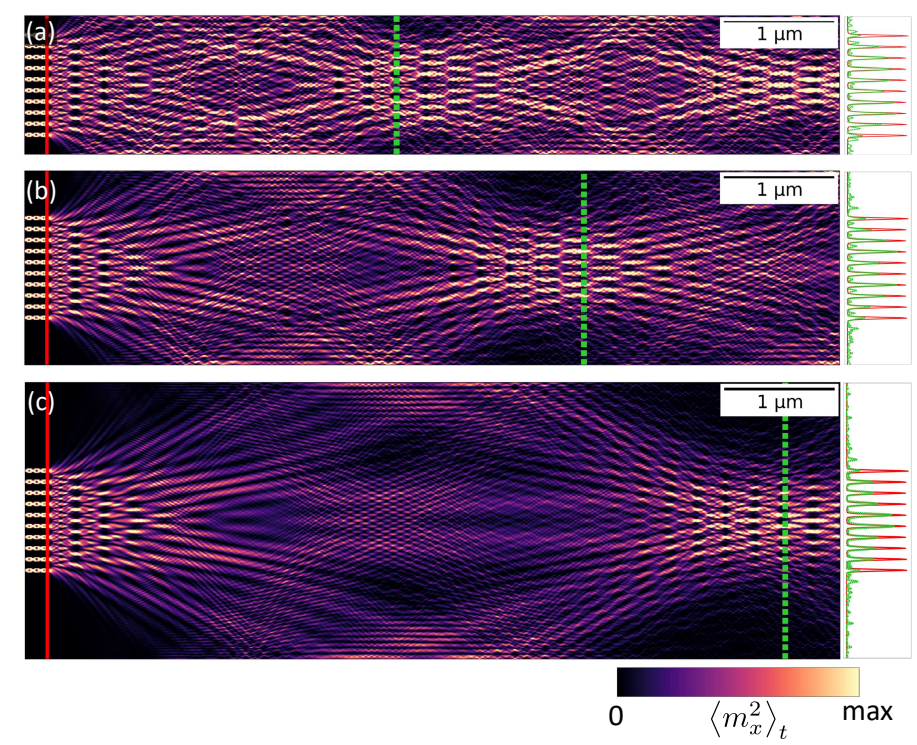

Figure 6. Intensity maps of SWs entering from a series of 10 singlemode $40 \mathrm{~nm}$ wide waveguides (on the left) into a (a) $1.25 \mu \mathrm{m}$, (b) $1.75 \mu \mathrm{m}$ and (c) $2.5 \mu \mathrm{m}$ wide multimode film. The distance between the sources is equal in all cases $100 \mathrm{~nm}$. The solid red and dashed green lines represent the cross-section along which the normalized SW intensities as a function of the width are plotted (on the right).

to transmit information about a geometry of a diffraction grating/array of sources over long distances thanks to the self-imaging phenomenon.

\section{IMPACT OF Gilbert DAMPING ON THE SELF-IMAGING EFFECT}

The Talbot effect in finite systems of thin ferromagnetic layers can be used in two ways. (i) At short distances from the source array, as we have presented in Fig. 4 for a wide distribution of a number of sources. Here the near diffraction field is distributed over the width of the multimode waveguide and is close to the classic Talbot carpet, and it doesn't significantly depend on the edges of the waveguide (see Figs. 36). (ii) As we presented in the other examples, where the near field pattern is reproduced over longer distances. Of course, especially the latter case raises the question of the SW damping and whether this effect is achievable experimentally.

On the basis of micromagnetic simulations, we analyzed the influence of a damping constant on the self-imaging effect, by simulations with the two values of $\alpha: 3.2 \times 10^{-4}$ [characteristic for yttrium iron garnet (YIG)], and $\alpha=$ $5 \times 10^{-3}$ (permalloy). The remaining material parameters, characteristic for permalloy, are unchanged in relation to the previous simulations. YIG and permalloy are some of the most popular magnetic materials in magnonics due to low damping; therefore, the choice the tabular damping values characteristic for these materials as an example is justified.

Figure 7 shows the SW amplitude distribution for two types of system geometries, with smaller separation between the inputs [(a) and (c)] and large separation [(b) and (d)], for the two values of $\alpha$. The conclusion that arises
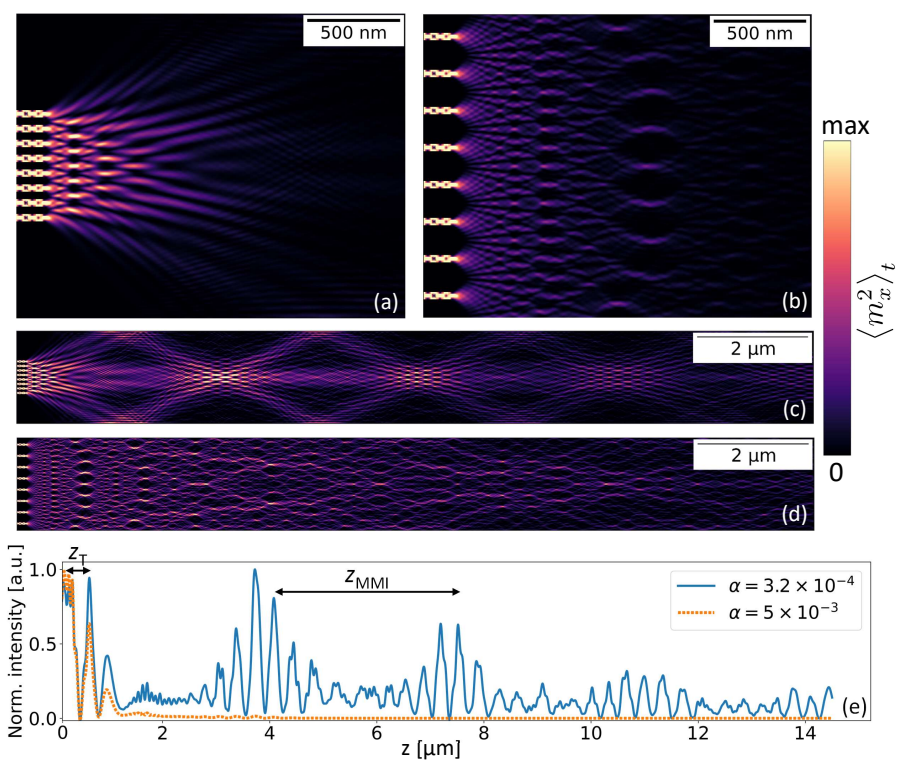

Figure 7. SW intensity maps in multimode waveguides with Gilbert damping factors equal to (a), (b) $\alpha=5 \times 10^{-3}$ and (c), (d) $\alpha=3.2 \times$ $10^{-4}$. The period of inputs in (a) and (c) is equal to $80 \mathrm{~nm}$, while in (b) and (d) is $200 \mathrm{~nm}$. In both cases the waveguide width is $1.64 \mathrm{\mu m}$. The graph (e) shows the intensity of SWs on a line from the fourth source for both analyzed damping values (and smaller input period cases). The Talbot length is marked as $z_{\mathrm{T}}$, while the length of reproducing self-images as $z_{\mathrm{MMI}}$.

is certainly a large decrease in the intensity of self-images in the far diffraction field for the $\alpha$ of permalloy - here detection by measuring techniques such as micro-BLS may be limited only to classic Talbot images formed near the source array. However, in systems with small damping, the situation is much more promising - reproductions at the distance of even a few $\mu \mathrm{m}$ from the sources should be measurable using standard techniques for measuring the dynamics of magnetization.

Figure 7(e) shows the SW intensity as a function of the distance from the array sources along the line crossing the fourth source. It can be clearly seen that in the system with the damping constant characteristic for permalloy (orange line) the decrease in SW energy by $50 \%$ occurs after around $700 \mathrm{~nm}$, thus only classic Talbot effect $\left(z_{\mathrm{T}}\right)$ can be exploited. For YIG-value of damping the signal decreases by $50 \%$ only after around $8 \mu \mathrm{m}$, which allows for observation at least 3 reproduced Talbot images $\left(3 z_{\mathrm{MMI}}\right)$ and makes it a good candidate for applications. It is also important to point out that both YIG, permalloy, and materials with a slightly higher damping factor can be used in systems where the Talbot effect in the near field will be used. This is especially promising for future potential magnonic devices where miniaturization, e.g. computing systems, will be a key.

\section{Conclusions}

The propagation of high-frequency SWs in multimode structures is of a great interest. Such studies allow understanding the dynamics of SWs in structures that can replace conventional CMOS systems in high-speed signal processing, logic circuits, and information storage technologies in 
the near future. Using different types of system geometry, materials and changing the direction and value of the external magnetic field, we can manipulate the interference field generated in the multimode part. SW self-imaging may be utilized to realize logic operations using interference patterns. Moreover, using the Talbot effect properties, similar systems can be used, for example, as input phase coherence controllers or (de)multiplexers.

The combination of the Talbot effect with MMI was the core of the structures proposed in this paper. We showed that a series of single-mode SW sources causes the formation of self-images in multimode waveguides in the near diffraction field and also to be transferred for much further distances creating reproduced Talbot carpets due to MMI. The quality of these reproduced carpets depends on the multimode waveguide widths, edges and the arrangement of single-mode waveguides. In materials with low Gilbert damping, this may result in focusing SW beams at far distances from the sources, while the near diffraction field may retain properties close to the theoretical undisturbed Talbot carpets even for moderate values of damping.

The out-of-plane alignment of the magnetization used in the paper is advantageous in the analysis of Talbot phenomena due to the circular shape of isofrequency contours. It makes the analyzed magnonic systems a good analogue of the systems known from photonics. However, there are still a number of technical issues, including high frequency and the value of the external magnetic field assumed in our simulations required to uniformly magnetize the system. One of the solutions may be the use of in-plane magnetized ferromagnetic layer, which would certainly contribute to lowering these two parameters, but at the same time introducing an anisotropy to the system.

We hope that this work will draw the attention of the magnonic community to the potential of SW self-imaging in many sorts of future technological solutions.

\section{ACKNOWLEDGMENT}

The research leading to these results has received funding from the National Science Centre of Poland, projects no. 2019/35/D/ST3/03729 and no. UMO2018/30/Q/ST3/00416. The simulations were partially performed at the Poznan Supercomputing and Networking Center (Grant No. 398).

\section{REFERENCES}

[1] A. G. Gurevich and G. A. Melkov, Magnetization oscillations and waves. CRC Press, Boca Raton, 1996.

[2] A. Barman, S. Mondal, S. Sahoo, and A. De, "Magnetization dynamics of nanoscale magnetic materials: A perspective," $J$. Appl. Phys., vol. 128, no. 17, p. 170901, 2020.

[3] A. Chumak, V. Vasyuchka, A. Serga, and B. Hillebrands, "Magnon spintronics," Nat. Phys., vol. 11, no. 6, pp. 453-461, 2015.

[4] V. E. Demidov and S. O. Demokritov, "Magnonic waveguides studied by microfocus Brillouin light scattering," IEEE Transactions on Magnetics, vol. 51, no. 4, pp. 1-15, 2015.

[5] Q. Wang, B. Heinz, R. Verba, M. Kewenig, P. Pirro, M. Schneider, T. Meyer, B. Lägel, C. Dubs, T. Brächer, and A. V. Chumak, "Spin pinning and spin-wave dispersion in nanoscopic ferromagnetic waveguides," Phys. Rev. Lett., vol. 122, p. 247202, Jun 2019.
[6] U. Garlando, Q. Wang, O. Dobrovolskiy, A. Chumak, and F. Riente, "Numerical model for 32-bit magnonic ripple carry adder," arXiv preprint arXiv:2109.12973, 2021.

[7] F. Heussner, M. Nabinger, T. Fischer, T. Brächer, A. A. Serga, B. Hillebrands, and P. Pirro, "Frequency-division multiplexing in magnonic logic networks based on caustic-like spin-wave beams," physica status solidi (RRL)-Rapid Research Letters, vol. 12, no. 12 , p. $1800409,2018$.

[8] F. Heussner, G. Talmelli, M. Geilen, B. Heinz, T. Brächer, T. Meyer, F. Ciubotaru, C. Adelmann, K. Yamamoto, A. A. Serga et al., "Experimental realization of a passive gigahertz frequency-division demultiplexer for magnonic logic networks," physica status solidi (RRL)-Rapid Research Letters, vol. 14, no. 4, p. 1900695, 2020.

[9] J. Gräfe, P. Gruszecki, M. Zelent, M. Decker, K. Keskinbora, M. Noske, P. Gawronski, H. Stoll, M. Weigand, M. Krawczyk, C. H. Back, E. J. Goering, and G. Schütz, "Direct observation of spin-wave focusing by a Fresnel lens," Phys. Rev. B, vol. 102, p. 024420, Jul 2020.

[10] Á. Papp, W. Porod, Á. I. Csurgay, and G. Csaba, "Nanoscale spectrum analyzer based on spin-wave interference," Scientific Reports, vol. 7, no. 1, pp. 1-9, 2017.

[11] Á. Papp, M. Kiechle, S. Mendisch, V. Ahrens, L. Sahin, L. Seitner, W. Porod, G. Csaba, and M. Becherer, "Experimental demonstration of a concave grating for spin waves in the Rowland arrangement," Scientific Reports, vol. 11, no. 1, pp. 1-8, 2021.

[12] A. Papp, W. Porod, and G. Csaba, "Nanoscale neural network using non-linear spin-wave interference," arXiv preprint arXiv:2012.04594, 2020.

[13] A. Papp, G. Csaba, and W. Porod, "Characterization of nonlinear spin-wave interference by reservoir-computing metrics," Applied Physics Letters, vol. 119, no. 11, p. 112403, 2021.

[14] Q. Wang, A. V. Chumak, and P. Pirro, "Inverse-design magnonic devices," Nature Communications, vol. 12, no. 1, pp. 1-9, 2021.

[15] D. Prychynenko, M. Sitte, K. Litzius, B. Krüger, G. Bourianoff, M. Kläui, J. Sinova, and K. Everschor-Sitte, "Magnetic skyrmion as a nonlinear resistive element: a potential building block for reservoir computing," Physical Review Applied, vol. 9, no. 1, p. 014034, 2018.

[16] H. Talbot, "Lxxvi. facts relating to optical science. no. iv," The London, Edinburgh, and Dublin Philosophical Magazine and Journal of Science, vol. 9, no. 56, pp. 401-407, 1836.

[17] L. Rayleigh, "On copying diffraction gratings and on some phenomenon connected therewith," Phil. Mag., vol. 11, p. 196, 1881.

[18] J. Wen, Y. Zhang, and M. Xiao, "The Talbot effect: recent advances in classical optics, nonlinear optics, and quantum optics," Adv. Opt. Photon., vol. 5, no. 1, pp. 83-130, Mar 2013.

[19] A. Bravin, P. Coan, and P. Suortti, "X-ray phase-contrast imaging: from pre-clinical applications towards clinics," Physics in Medicine and Biology, vol. 58, no. 1, pp. R1-R35, 2012.

[20] M. Gołębiewski, P. Gruszecki, M. Krawczyk, and A. E. Serebryannikov, "Spin-wave Talbot effect in a thin ferromagnetic film," Phys. Rev. B, vol. 102, p. 134402, Oct 2020.

[21] X. Wang, H. Chen, H. Liu, L. Xu, C. Sheng, and S. Zhu, "Selffocusing and the Talbot effect in conformal transformation optics," Phys. Rev. Lett., vol. 119, p. 033902, Jul 2017.

[22] A. Vansteenkiste, J. Leliaert, M. Dvornik, M. Helsen, F. GarciaSanchez, and B. Van Waeyenberge, "The design and verification of mumax3," AIP Advances, vol. 4, no. 10, p. 107133, 2014. 